\title{
DETECCIÓN DE PATRONES DE COMPORTAMIENTO HUMANO EN AMBIENTES INTELIGENTES: UN MAPEO SISTEMÁTICO DE FUENTES SECUNDARIAS
}

\author{
María Luisa Córdoba-Tlaxcalteco y Edgard Benítez-Guerrero \\ Universidad Veracruzana, México
}

\begin{abstract}
RESUMEN
En este trabajo se realiza un mapeo sistemático en fuentes secundarias de la literatura sobre el problema de detección automática de comportamiento humano en ambientes inteligentes. El contexto donde se presenta el problema es el área de Inteligencia Ambiental, que se dirige a tener espacios tecnológicamente enriquecidos que apoyen proactivamente a las personas en su vida diaria. Con el propósito de organizar la información recolectada, y presentarla en una forma sintetizada, se presenta una taxonomía con diferentes aspectos críticos del problema planteado, enfatizando los tipos de ambientes inteligentes, el grado de detalle en el nivel de representación de la figura humana y de su contexto, y las técnicas utilizadas para detectar comportamientos. El objetivo de éste trabajo es identificar áreas de oportunidad para la investigación en Inteligencia Ambiental.
\end{abstract}

PALABRAS CLAVE

Inteligencia Ambiental, Detección de Comportamientos, Mapeo Sistemático

\section{INTRODUCCIÓN}

Las sociedades humanas se desarrollan en entornos que son constantemente monitoreados para supervisar y mejorar la seguridad o el bienestar de sus integrantes (Bhardwaj 2018). La necesidad de automatizar los procesos de monitoreo y de detección de actividades humanas ha llevado a la creación del área de la Inteligencia Ambiental, término que se refiere a la parte de la Inteligencia Artificial dedicada a la generación de espacios físicos donde los seres humanos interactúan con tecnologías informáticas y de comunicación que le sirven de apoyo (Zhang 2016.). En este contexto, el reconocimiento automático de comportamientos humanos es un elemento crítico, ya que el sistema informático necesariamente debe conocer lo que sucede dentro del ambiente, a fin de responder o actuar ante ello.

El objetivo del presente trabajo es analizar el estado actual de los trabajos sobre el reconocimiento automático de comportamiento humano en ambientes inteligentes, así como identificar posibles líneas de investigación. Para ello realizamos un mapeo sistemático de fuentes secundarias. El método seguido para su elaboración se describe en la Sección 2. Los temas tratados por los trabajos consultados se distinguen en la Sección 3. Finalmente, se concluye en la Sección 4. Los resultados son un trabajo previo a una revisión exhaustiva en fuentes primarias, en donde se pretende analizar y clasificar documentos recientes sobre la detección de comportamientos con énfasis en modelos semánticos.

\section{MÉTODO PARA LA REVISIÓN SISTEMÁTICA DE LA LITERATURA}

Un mapeo sistemático es un proceso de clasificación que permite el análisis cuantitativo de las diferentes clases o áreas de un determinado tema en la literatura. Este procedimiento se lleva a cabo en tres fases: i) definición de las preguntas de investigación, ii) búsqueda de documentos y iii) selección de documentos para inclusión o exclusión (Petersen 2015). A continuación, se describe la forma en que instanciamos este procedimiento para este trabajo. 


\subsection{Definición de las Preguntas de Investigación}

La definición de las preguntas de investigación (Tabla 1) permite recuperar información relevante y una visión general del tema de interés. Con la intención de no forzar la búsqueda hacia un tema específico, se determina una cadena de búsqueda con los sinónimos de las palabras generales en inglés "behaviour", "ambient intelligence", y "survey". La cadena de búsqueda fue: (ambient or environment or space) and (smart or intelligent) and (behavior or behaviour) and (review or survey).

Tabla 1. Preguntas de investigación

P1 ¿En qué tipos de ambientes inteligentes se realiza el reconocimiento?

P2 ¿Qué representación del ambiente se utiliza en el reconocimiento?

P3 ¿Qué enfoques son usados para el procesamiento de la información del ambiente?

P4 ¿Qué tipos de comportamiento humano es reconocidos en la literatura?

\subsection{Búsqueda de Documentos}

Se analizaron documentos recientes en cuatro bases de datos científicas: Springer Link (link.springer.com), IEEE Xplore Digital Library (ieeexplore.ieee.org), ACM Digital Library (dl.acm.org) y ScienceDirect (sciencedirect.com). Ver Tabla 3.

\subsection{Selección de Documentos para Inclusión y Exclusión}

Los artículos que nos interesa incluir en este mapeo son publicaciones con relevancia para responder las preguntas de investigación. En consecuencia, es necesario establecer criterios de inclusión y exclusión, ver Tabla 2.

Tabla 2. Criterios de inclusión y exclusión

\begin{tabular}{ll}
\hline Inclusión & Exclusión \\
\hline Revisiones de la literatura. & No son revisiones de la literatura. \\
Se analiza la detección de comportamiento. & No se analiza la detección de \\
Publicados en el periodo 2010-2020. & comportamientos. \\
Escritos en español o inglés. & Publicaciones anteriores al 2010. \\
Artículos de revistas o documentos de & No están escritos en español o inglés. \\
conferencias. & Presentaciones de PowerPoint o similares. \\
\hline
\end{tabular}

Tabla 3. Documentos clasificados por base de datos científicas

\begin{tabular}{|l|l|l|l|l|}
\hline & Springer & IEEE & ACM & SCIENCE DIRECT \\
\hline Resultado de la búsqueda & 285 & 36 & 165 & 76 \\
\hline $\begin{array}{l}\text { Al aplicar los criterios de } \\
\text { inclusión y exclusión }\end{array}$ & 10 & 5 & 2 & 1 \\
\hline
\end{tabular}

\subsection{Análisis de la Información y Síntesis en una Taxonomía}

Las preguntas de investigación en la Tabla 1 inducen una taxonomía u ordenamiento de los aspectos que en este trabajo son destacados como cruciales para la detección de comportamiento, y reflejan un aspecto crítico a considerar en el análisis y comparación de los diferentes trabajos sobre detección automática de comportamientos. La Figura 1 a continuación muestra en forma de esquema las variantes en cada aspecto. 


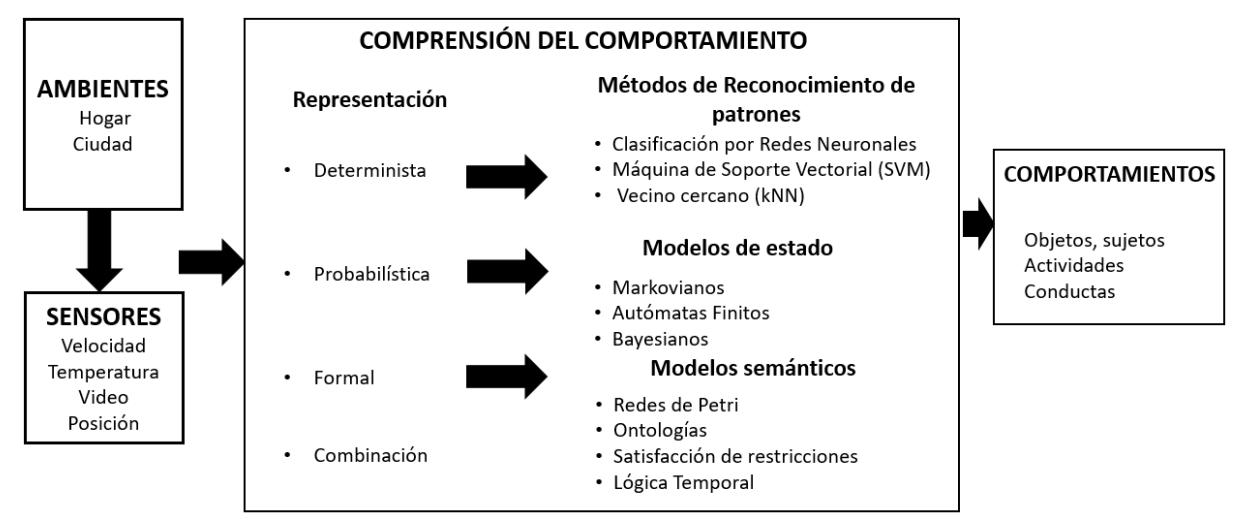

Figura 1. Esquema con los aspectos del problema de detección automática de comportamiento humano a revisar en la literatura

\section{RESULTADOS}

A continuación, se presentan los resultados obtenidos de acuerdo al esquema en la Figura 1.

\subsection{Ambientes Inteligentes}

La identificación del tipo de ambiente inteligente es crucial, porque además de establecer el espacio físico de desarrollo de los comportamientos humanos, también determina en gran medida la aplicabilidad y la relevancia de la detección (Fahim 2019). Mientras que el desarrollo de sistemas de reconocimiento de actividades en casas, oficinas, o aulas inteligentes conllevan la generación de asistencia automática en la vida diaria de personas con menor o mayor grado de necesidad o vulnerabilidad (Vallabh 2018), otros trabajos están orientados a la organización de poblaciones humanas más complejas como las ciudades inteligentes, aeropuertos, estaciones de transporte urbano, etc. (Zhang 2016). El grado de complejidad del ambiente inteligente es muy importante, porque la automatización del reconocimiento de los comportamientos es un reto más grande en los ambientes complejos, que han sido recientemente abordados (Fahim 2019, Rodríguez 2014).

\subsection{Sensores}

El ambiente inteligente está caracterizado por las variables como la ocupación de personal en un espacio, y es por medio de un conjunto de sensores que se adquieren las características fundamentales del entorno físico. Éstas pueden ser físicas (velocidad, aceleración, temperatura), visuales (imágenes, video), espaciales (GPS, posición), biométricas (cardiografía, frecuencia respiratoria), y otras. De acuerdo al tipo de información que los sensores capturan, se puede clasificar a la red de sensores en dos tipos: biométricos, e video o combinación de ellos (Lowe 2014, Patel 2012). La fusión de diferentes tipos de datos es bastante común hoy en día.

\subsection{Reconocimiento del Comportamiento}

Una vez que se realiza el sensado del ambiente, el siguiente aspecto a considerar es la forma en que se organizan o procesan los datos, imágenes, videos, o cualquier otro tipo de registro. En este punto se distingue como primer punto la representación o abstracción de los registros, clasificando ésta en representaciones deterministas o no deterministas, como en Aprendizaje no Supervisado (donde no hay a priori una estructura en ellos (Fahim 2019), representaciones basadas en conocimiento y razonamiento, que asumen la existencia a priori de reglas lógicas que permiten inferir formalmente la presencia de actividades o comportamientos (Zhang 2016), o representaciones que toman en cuenta la incertidumbre inherente al reconocimiento de comportamiento, usando la Probabilidad como medida cuantitativa de esta incertidumbre (Zhu 2017). El siguiente aspecto, íntimamente relacionado con la representación del ambiente, es la técnica. Aquí se distinguen técnicas basadas en el concepto dinámico de estado, como son los modelos markovianos o los autómatas finitos (Amiribesheli 2015, Borazio2013), el procesamiento transformacional que considera a los datos como una imagen estática del ambiente (usando técnicas de Reconocimiento de Patrones, como Máquinas de soporte vectorial o Redes 
Neuronales (Lussier 2019), y el procesamiento basado en el uso de etiquetas semánticas, el cual permite introducir a un lenguaje como elemento estructural del modelo. En este último tipo de procesamiento también es notable distinguir el uso de lenguajes gráficos o lógicos, como las Redes de Petri o las Ontologías (Rodríguez 2017).

\subsection{Tipos de Comportamiento}

En la literatura se identificaron diferentes grados de reconocimiento de comportamientos, desde nivel básico, intermedio y complejo. En lo básico se encuentra el reconocimiento de objetos (armas, objetos de trabajo, etc.), de sujetos (caras, silueta de personas o animales), o de cualquier otra característica elemental del ambiente (Zhu 2017). En un nivel intermedio se encuentra la detección de actividades entendidas como acciones desarrolladas en un corto intervalo de tiempo (deportes, actividades del hogar, anomalías, etc.), (Patel 2012). En el nivel complejo se encuentra el reconocimiento de patrones o tendencias en la realización de actividades, así como la detección de interacciones entre varias personas (Rohit 2017). La detección de un patrón de actividades o interacciones conlleva la comprensión de intencionalidad (como una persona que patea a otra para golpearla), la identificación de conjuntos de personas interactuando para realizar algo en grupo (como el control de multitudes), o la regularidad estadística de actividades a lo largo del tiempo (Fahim 2019).

En la Tabla 4 se resume la información obtenida al realizar el mapeo sistemático.

Tabla 4. Resultado de la búsqueda

\begin{tabular}{|c|c|c|c|c|}
\hline \multirow{2}{*}{ DOCUMENTO } & \multirow{2}{*}{ AMBIENTE } & \multirow{2}{*}{ SENSORES } & \multicolumn{2}{|c|}{ COMPRENSIÓN DEL COMPORTAMIENTO } \\
\hline & & & REPRESENTACIÓN & TÉCNICA \\
\hline $\begin{array}{l}\text { Amiribesheli, } \\
\text { M. et al, } 2015 .\end{array}$ & Hogar & $\begin{array}{l}\text { Físicos, } \\
\text { espaciales, y } \\
\text { visuales }\end{array}$ & $\begin{array}{l}\text { Determinista, } \\
\text { probabilista y formal }\end{array}$ & $\begin{array}{l}\text { Árboles de decisión, lógica difusa, redes } \\
\text { neuronales artificiales, máquinas soporte } \\
\text { vectorial, clasificador Naive Bayes, } \\
\text { modelos ocultos de Markov, patrones } \\
\text { emergentes y ontologías. }\end{array}$ \\
\hline $\begin{array}{l}\text { Borazio, M. et } \\
\text { al, } 2013 .\end{array}$ & Hogar & $\begin{array}{l}\text { Wearables } \\
\text { físicos }\end{array}$ & Probabilística & Modelos ocultos de Markov \\
\hline $\begin{array}{l}\text { Fahim, M. et al, } \\
2019 .\end{array}$ & $\begin{array}{l}\text { No } \\
\text { específico }\end{array}$ & $\begin{array}{l}\text { Visuales y } \\
\text { Físicos }\end{array}$ & $\begin{array}{l}\text { Determinista } \\
\text { Y probabilística }\end{array}$ & $\begin{array}{l}\text { Métodos estadísticos, modelos de } \\
\text { aprendizaje automático y modelos de } \\
\text { aprendizaje profundo. }\end{array}$ \\
\hline $\begin{array}{l}\text { Lowe, } S . \text { A. et } \\
\text { al, } 2014 .\end{array}$ & Hogar & $\begin{array}{l}\text { Físicos, } \\
\text { espaciales, } \\
\text { visuales } \\
\end{array}$ & No especificado & No especificado \\
\hline $\begin{array}{l}\text { Lussier, M. et } \\
\text { al, } 2019 .\end{array}$ & Hogar & $\begin{array}{l}\text { Espaciales, } \\
\text { visuales }\end{array}$ & Determinista & Aprendizaje supervisado y no supervisado \\
\hline $\begin{array}{l}\text { Mahata, J., et } \\
\text { al, } 2017\end{array}$ & Varios & Video & Determinista & $\begin{array}{l}\text { Red neuronal artificial, los modelos } \\
\text { bayesianos, el modelo oculto de markov, el } \\
\text { modelo de mezcla gaussiana, la máquina de } \\
\text { vectores de soporte y el aprendizaje } \\
\text { profundo }\end{array}$ \\
\hline $\begin{array}{l}\text { Patel, S. et al, } \\
2012 .\end{array}$ & Hogar & $\begin{array}{l}\text { Wearables y } \\
\text { ambientales }\end{array}$ & No específicado & No específicado \\
\hline $\begin{array}{l}\text { Rodríguez, } N . \\
\text { D.et al, } 2014 .\end{array}$ & Hogar & Varios & Formal & Modelos lógicos formales, razonamiento \\
\hline $\begin{array}{l}\text { Rohit, K. et al, } \\
2017 .\end{array}$ & Ciudad & Video & Determinista & $\begin{array}{l}\text { Enfoque basado en objetos y enfoque } \\
\text { basado en holística }\end{array}$ \\
\hline $\begin{array}{l}\text { Vallabh, P. et } \\
\text { al, } 2018 .\end{array}$ & Hogar & Wearables & Formal & $\begin{array}{l}\text { Basados en reglas y aprendizaje } \\
\text { automático. }\end{array}$ \\
\hline $\begin{array}{l}\text { Zaidan, A. et al, } \\
\text { 2018. }\end{array}$ & Hogar & $\begin{array}{l}\text { Video, } \\
\text { espaciales }\end{array}$ & Determinista & Minería de datos \\
\hline $\begin{array}{l}\text { Zhang, C, et al, } \\
2016 .\end{array}$ & Ciudad & Varios & Probabilístico & Análisis Estadístico \\
\hline
\end{tabular}




\section{CONCLUSIÓN}

De acuerdo con los documentos recuperados, los temas dominantes en el área son la aplicación de detección de comportamientos humanos en ambientes de la vida diaria, como hogares (67\%) y ciudades (17\%). En cuanto a los tipos extracción de información de entorno destaca el uso de wearables (25\%) y video (17\%). De acuerdo a la finalidad de realizar el reconocimiento, sobresale la vida asistida y salud (25\%), el monitoreo de actividades de la vida diaria $(25 \%)$ y detección de anomalías (25\%). Respecto a las técnicas para la comprensión de comportamientos, se observa que la mayoría son con relación a los métodos de reconocimiento de patrones y modelos de estados (75\%), y una minoría incluye en su taxonomía a los modelos semánticos (25\%). En general se observa la tendencia de integrar tecnologías en software y hardware para crear ambientes inteligentes muy controlados. Existe el reto de extrapolar estos resultados a sistemas abiertos donde intervienen aspectos no controlados, como la agricultura inteligente, o en ambientes complejos ya sea por su dimensión (como las ciudades inteligentes), o por la complejidad de su interacción (como las aulas inteligentes). Naturalmente, hay aspectos que no fueron abordados en este mapeo sistemático. Por ejemplo la administración automática de cantidades masivas de datos o imágenes (Big Data, y las alternativas para el almacenamiento masivo), la reactividad del reconocimiento a cambios de comportamiento o contexto (aprendizaje incremental, lógica temporal), por mencionar solo algunos puntos.

\section{AGRADECIMIENTO}

Este trabajo fue apoyado parcialmente por el Consejo Nacional de Ciencia y Tecnología (CONACYT) de México en el marco del proyecto de Cátedras "Infraestructura para Agilizar el Desarrollo de Sistemas Centrados en el Usuario" (Ref. 3053). Agradecemos también a CONACYT por la beca de Doctorado número 785914 del primer autor, así como a la Universidad Veracruzana por el apoyo para el desarrollo de esta investigación.

\section{REFERENCIAS}

Amiribesheli, M. (2015). A review of smart homes in healthcare. Journal of Ambient Intelligence and Humanized Computing, 6(4), 495-517. SPRINGER

Borazio, M. (2013). Improving activity recognition without sensor data: a comparison study of time use surveys. In Proceedings of the 4th Augmented Human International Conference (pp. 108-115). ACM.

Fahim, M. (2019). Anomaly Detection, Analysis and Prediction Techniques in IoT Environment: A Systematic Literature Review. IEEE Access, 7, 81664-81681. IEEE.

Gayathri, K. S. (2017). Probabilistic ontology based activity recognition in smart homes using Markov Logic Network. Knowledge-Based Systems, 121, 173-184. ACM

Lowe, S. A. (2014). Monitoring human health behaviour in one's living environment: a technological review. Medical engineering \& physics, 36(2), 147-168. SCIENCE DIRECT

Lussier, M. (2019). Early Detection of Mild Cognitive Impairment With In-Home Monitoring Sensor Technologies Using Functional Measures: A Systematic Review. IEEE journal of biomedical and heal th informatics, 23(2), 838-847.

Mahata, J., (2017). Recent advances in human behaviour understanding: A survey. In 2017 Devices for Integrated Circuit (DevIC) (pp. 751-755). IEEE.

Novitzky, P. (2015). A review of contemporary work on the ethics of ambient assisted living technologies for people with dementia. Science and engineering ethics, 21(3), 707-765. SPRINGER

Patel, S. (2012). A review of wearable sensors and systems with application in rehabilitation. Journal of neuroengineering and rehabilitation, 9(1), 21. SPRINGER

Petersen, K. (2015). Guidelines for conducting systematic mapping studies in software engineering: An update. Information and Software Technology, 64, 1-18. SCIENCE DIRECT

Rodríguez, N. D. (2014). A survey on ontologies for human behavior recognition. ACM Computing Surveys (CSUR), 46(4), 43. ACM

Rohit, K. (2017). A review on abnormal crowd behavior detection. En Conferencia Internacional sobre innovaciones en sistemas de información, embebidos y Comunicación 2017 (ICIIECS) (pp. 1-3). IEEE.

Vallabh, P. (2018). Fall detection monitoring systems: a comprehensive review. Journal of Ambient Intelligence and Humanized Computing, 9(6), 1809-1833. SPRINGER

Zaidan, A. (2018). A review on intelligent process for smart home applications based on IoT: coherent taxonomy, motivation, open challenges, and recommendations. Artificial Intelligence Review, 1-25. SPRINGER

Zhang, C, (2016). A review of occupant behavior models in residential building: Sensing, modeling, and prediction. In 2016 Chinese Control and Decision Conference (CCDC) (pp. 2032-2037). IEEE. 